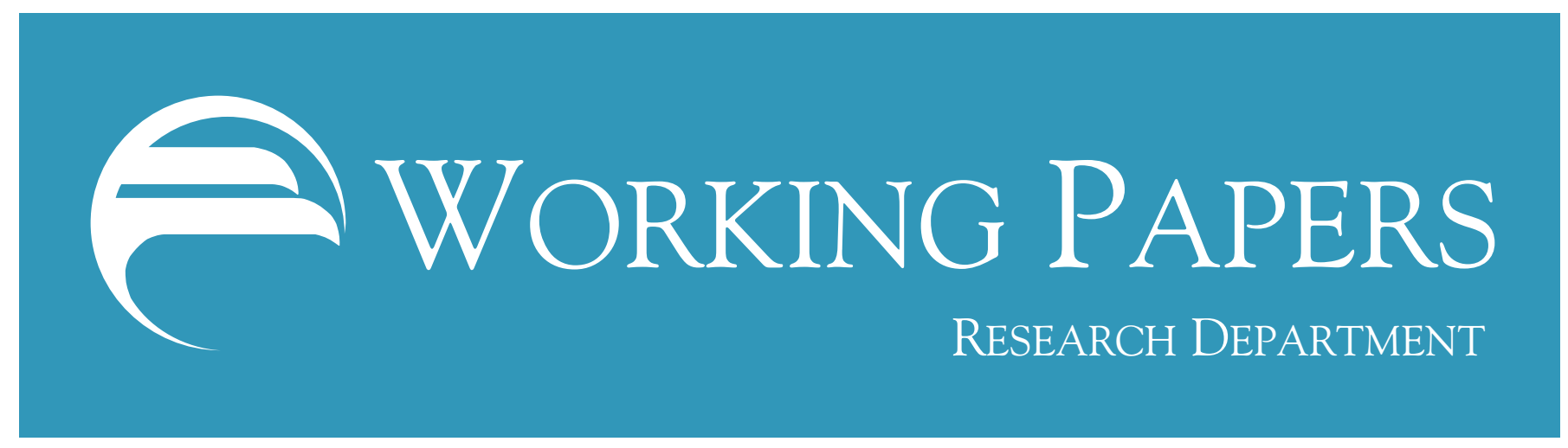

\title{
WORKING PAPER NO. 03-2 \\ THE EFFECTS OF A BABY BOOM ON STOCK PRICES AND CAPITAL ACCUMULATION IN THE PRESENCE OF SOCIAL SECURITY
}

Andrew B. Abel

The Wharton School, University of Pennsylvania and

National Bureau of Economic Research

July 2002

\section{FEDERAl ReserVe BANK OF PHILADELPHIA}




\title{
The Effects of a Baby Boom on Stock Prices and Capital Accumulation in the Presence of Social Security
}

\author{
Andrew B. Abel* \\ The Wharton School of the University of Pennsylvania \\ and \\ National Bureau of Economic Research
}

First version: September 1999; This version: July 2002

\begin{abstract}
Is the stock market boom a result of the baby boom? This paper develops an overlapping generations model in which a baby boom is modeled as a high realization of a random birth rate, and the price of capital is determined endogenously by a convex cost of adjustment. A baby boom increases national saving and investment and thus causes an increase in the price of capital. The price of capital is meanreverting so the initial increase in the price of capital is followed by a decrease. Social Security can potentially affect national saving and investment, though in the long run, it does not affect the price of capital.
\end{abstract}

Keywords: baby boom, Social Security, stock prices, Golden Rule

*Mailing Address: Department of Finance, The Wharton School of the University of Pennsylvania, 3620 Locust Walk, Philadelphia, PA 19104-6367. I thank Bill Dupor, Joao Gomes, Urban Jermann, Nick Souleles, Chris Telmer, and Amir Yaron for helpful discussions, the editor and three anonymous referees for helpful comments, and Arianna Degan for excellent research assistance. I also thank seminar participants at Columbia University, the Federal Reserve Banks of Minneapolis, New York, and Richmond, Iowa State University, Stanford University, University of California at Berkeley, University of Southern California, NBER Summer Institute, and the Penn Macro Lunch Group. 
As the baby boom generation progresses through middle age, with its oldest members anticipating retirement about a decade in the future, public attention has increasingly focused on the provision of income during retirement. Retirement income is built on three pillars: Social Security, private pensions, and other private saving. Although the Social Security system in the United States currently runs a surplus of more than $\$ 100$ billion per year, the enormous flow of benefits that will be paid to retired baby boomers will cause large deficits in a few decades. As a consequence, the solvency of the Social Security system is in peril. However, the prospects for private pensions appear optimistic due in part to the dramatic increase in stock prices during the 1980s and 1990s. At the upper end of the income distribution, the rise in stock prices has created "401(k) millionaires," and many workers of more modest means have also benefited from the boom in stock prices. Other private saving has also benefited from the run-up in the stock market.

As the stock market continued to rise sharply through the late 1990s, many observers expressed concern that the market was overvalued and that the high value of stock prices reflected a bubble. At least some of the concern about a bubble appeared justified by the tumble in stock prices, especially in the prices of technology stocks, that began in March 2000. However, even after falling to a level in July 2002 that was slightly more than $40 \%$ below its peak, the stock market remained more than 8 times its level at the beginning of $1980 .{ }^{1}$ In this paper, I examine the long-term sustained increase in the value of the stock market over the period since 1980 . Instead of trying to determine whether there was (or still is) a bubble in the stock market, I will pursue a more modest and focused goal in this paper. Specifically, I will use the discipline of a general equilibrium model with overlapping generations of rational consumers to examine the logical consistency of the argument that the flow of private saving by middle-aged baby boomers helped fuel the booming stock market. Potentially offsetting the argument that purchases of stocks by middle-aged baby boomers is driving up stock prices is a similar argument that these investors will eventually sell large amounts of stock during retirement, thereby causing a decline in stock prices. And if these investors are forward-looking in the first place, would they so eagerly buy

\footnotetext{
${ }^{1}$ Stock prices are measured here by the S\&P 500 index. This index rose by a factor of 1.54 during the 1960s, 1.17 during the 1970s, 3.27 during the 1980s, and 4.16 during the 1990s. Comparing 20-year periods, this index rose by a factor of 1.80 over the $1960 \mathrm{~s}$ and 1970 s, and by a factor of 13.61 over the 1980s and 1990s. On July 30, 2002, the S\&P 500 index was 8.36 times as high as its value on December 31, 1979.
} 
stocks that are destined to fall in price eventually?

A natural framework for analyzing the effects on stock prices and capital accumulation of a baby boom is an overlapping generations model with production and capital accumulation as in Diamond (1965). However, I need to modify the classic Diamond model in two ways. First, population grows at a constant rate in the Diamond model, so I must modify the model to allow for variation in the population growth rate. I will model the birth rate as an i.i.d. random variable. The second modification reflects the goal of analyzing the behavior of the price of capital. In the Diamond model, as in most neoclassical growth models, the price of capital in terms of consumption is immutably equal to one because a unit of output can be used either for one unit of consumption or to create one unit of capital. To make the price of capital endogenous, I assume that there is a convex adjustment cost technology for converting consumption goods into capital goods.

Modifying the Diamond model to incorporate adjustment costs and random population growth leads to a model that can be used to examine the effect of a baby boom on stock prices and capital accumulation. ${ }^{2}$ I also include a Social Security system in the model and I allow (but do not require) the Social Security system to accumulate capital in a trust fund. One reason for including a Social Security system is that in an overlapping generations model with consumers who live for two periods, and have no bequest motive, private saving is motivated only by the desire to consume during retirement. To the extent that Social Security provides a substantial portion of retirement consumption for many consumers, I want to take account of Social Security when analyzing the saving behavior of individuals. An additional reason for including Social Security is that it provides a set of fiscal policy tools that can potentially affect consumption, capital accumulation, and stock prices. Indeed, Social Security policy can be used to allow the economy to attain the Golden Rule in the long run.

I describe the production and adjustment cost technologies in Section 1. In Section 2, I introduce the Social Security system. The specification of the Social Security system is flexible enough to include a pure pay-as-you-go system or a system with a trust fund. I model the behavior of individual consumers in Section 3. In Section 4, I derive the equilibrium values of

\footnotetext{
${ }^{2}$ Bohn (1999) presents a version of the Diamond model with uncertain birth rates, but the price of capital is immutably fixed at unity, so that model cannot be used to analyze the effects of a baby boom on stock prices.
} 
the capital-labor ratio and the price of capital. I show that a baby boom increases the price of capital, and that the price of capital reverts toward its mean in the following period.

An important variable characterizing capital accumulation is the investmentoutput ratio. In the model presented here, the investment-output ratio is constant in the long run, even though shocks to technology and the birth rate cause stochastic variation in the capital-labor ratio and the price of capital in the long run. Two interesting baseline cases in which to study the investment-output ratio are a laissez-faire economy, which I analyze in Section 5, and an economy that has attained the Golden Rule, which I analyze in Section 6. Also in Section 6, I analyze the dynamic efficiency or inefficiency of an economy with costs of adjusting the capital stock, and I analyze policies to attain the Golden Rule. Sections 7 and 8 analyze the long-run behavior of the investment-output ratio for different classes of Social Security policies. Section 7 examines defined-benefit policies with a constant replacement rate and a trust fund that is proportional to the aggregate capital stock. Section 8 examines defined-contribution Social Security systems. I present concluding remarks in Section 9.

\section{Production Technologies}

Consider an overlapping generations closed economy in which consumers live for two periods. Each consumer supplies one unit of labor when young and does not work when old. This economy has two production technologies. The first production technology uses capital and labor to produce a consumption good. The consumption good can be consumed in the period in which it is produced, or it can be used as an input to the second technology, the capital adjustment technology, which uses the consumption good and capital to produce capital for use in the following period.

The consumption good technology is

$$
Y_{t}=A_{t} K_{t}^{\alpha} N_{t}^{1-\alpha}
$$

where $0<\alpha<1, Y_{t}$ is the gross production of consumption goods before diverting some of these goods to the capital adjustment technology, $K_{t}$ is the aggregate capital stock at the beginning of period $t, N_{t}$ is the aggregate input of labor in period $t$, and $A_{t}>0$ is total factor productivity $(T F P)$. 
Total factor productivity follows a geometric random walk

$$
\ln A_{t}=\ln A_{t-1}+\varepsilon_{A, t}
$$

where the growth rate of $T F P, \varepsilon_{A, t}$, is i.i.d.

The output of the consumption goods technology is used for two purposes: consumption and capital formation. Let $C_{t}$ be the aggregate consumption of all consumers (young and old) during period $t$, and let $I_{t}$ be the aggregate amount of consumption goods diverted from consumption and used as an input to the capital adjustment technology. Therefore,

$$
Y_{t}=C_{t}+I_{t} .
$$

The capital adjustment technology uses consumption goods and the current capital stock to produce capital available for use in the following period. This technology is given by $K_{t+1}=G\left(K_{t}, I_{t}\right)$ where $G\left(K_{t}, I_{t}\right)$ is nondecreasing and linearly homogenous in $K_{t}$ and $I_{t}$. Convex adjustment costs are represented by the restriction $\frac{\partial^{2} G}{\partial I_{t}^{2}}<0$. The most natural specification for $G\left(K_{t}, I_{t}\right)$ is as

$$
G\left(K_{t}, I_{t}\right)=g\left(K_{t}, I_{t}\right)+(1-\delta) K_{t}
$$

where $g\left(K_{t}, I_{t}\right)$ represents gross investment and is non-decreasing and linearly homogeneous in $K_{t}$ and $I_{t}, \frac{\partial^{2} g}{\partial I_{t}^{2}}<0$, and $0 \leq \delta \leq 1$ is the depreciation rate. Net investment, $K_{t+1}-K_{t}$, equals gross investment minus depreciation, $\delta K_{t}$.

Instead of equation (4), I will adopt the following log-linear specification ${ }^{3}$ of $G\left(K_{t}, I_{t}\right)$

$$
K_{t+1}=G\left(K_{t}, I_{t}\right) \equiv a I_{t}^{\phi} K_{t}^{1-\phi}
$$

where $0 \leq \phi \leq 1$ and $a>0 .{ }^{4}$ I chose the log-linear specification in equation (5) because, as discussed in Section 4, this specification helps to simplify

\footnotetext{
${ }^{3}$ Basu (1987) uses the adjustment cost function in equation (5) with $a=1$ and examines equilibrium asset prices. Because Basu's model has a representative infinitely-lived consumer, it cannot be used to examine the impact of a baby boom on stock prices.

${ }^{4}$ If $\phi=1$ and $a=1$, equation (5) is simply $K_{t+1}=I_{t}$, which is the capital accumulation equation in the neoclassical growth model with complete depreciation of capital in each period. If $\phi=0$ and $a=1$, the capital accumulation equation in (5) is $K_{t+1}=K_{t}$, so that the capital stock is constant, as in the Lucas (1978) fruit-tree model of asset pricing.
} 
the behavior of the equilibrium price and quantity of capital. The analytic tractability resulting from the loglinear specification in equation (5) is achieved at the cost of violating the linear relationship among gross investment, net investment, and depreciation in equation (4), except in the extreme case in which capital fully depreciates within one period so that $\delta=1$. When $\delta<1$, the specification in equation (5) is, at best, an approximation of the more appealing specification in equation (4).

The price of capital is determined by the capital adjustment technology. Define $q_{t}$ to be the price, in terms of consumption goods, of acquiring one unit of capital at the end of period $t$ to be carried into period $t+1$. This price is the amount that $I_{t}$ must be increased to increase $K_{t+1}$ by one unit, which is $\left(\frac{d K_{t+1}}{d I_{t}}\right)^{-1}$. Thus, equation (5) implies

$$
q_{t}=\frac{1}{a \phi}\left(\frac{I_{t}}{K_{t}}\right)^{1-\phi}
$$

If $0<\phi<1$, the price of capital is an increasing function of the investmentcapital ratio, $\frac{I_{t}}{K_{t}}$. In the neoclassical growth model $a=1$ and $\phi=1$, so equation (6) indicates that the price of capital is constant and equal to one.

The value, measured in terms of current consumption goods, of the aggregate capital stock carried into period $t+1$ is $q_{t} K_{t+1}$. Multiplying both sides of equation (6) by $K_{t+1}$ and using the capital adjustment technology in equation (5) yields

$$
q_{t} K_{t+1}=\frac{1}{\phi} I_{t}
$$

The services of capital and labor are rented in competitive factor markets so that each factor earns its marginal product. Thus, the wage rate per unit of labor is

$$
w_{t}=(1-\alpha) \frac{Y_{t}}{N_{t}}
$$

Capital earns a rental in both technologies. The rental earned by capital in the production of consumption goods is

$$
\nu_{t}^{C}=\alpha \frac{Y_{t}}{K_{t}}
$$

The rental earned by capital in the capital adjustment technology, $\nu_{t}^{K}$, equals the marginal product of capital in this technology (measured in terms of 
capital in the following period) multiplied by the relative price of capital, $q_{t}$. It follows from equations (5) and (6) that

$$
\nu_{t}^{K}=\frac{1-\phi}{\phi} \frac{I_{t}}{K_{t}}
$$

The total rental to capital during period $t$ is the sum of the rentals $\nu_{t}^{C}$ and $\nu_{t}^{K}$. Since a unit of capital used in period $t$ was purchased at a price of $q_{t-1}$ at the end of period $t-1$, the rate of return to capital is

$$
R_{t}=\frac{\nu_{t}^{C}+\nu_{t}^{K}}{q_{t-1}} .
$$

An alternative expression for the rate of return on capital can be obtained by substituting equations (9) and (10) for $\nu_{t}^{C}$ and $\nu_{t}^{K}$, respectively, into equation (11) to obtain

$$
R_{t}=\frac{\alpha Y_{t}+\frac{1-\phi}{\phi} I_{t}}{q_{t-1} K_{t}} .
$$

Gross domestic product, GDP, equals the market value of final consumption goods, $C_{t}$, plus the market value of capital to be carried into the following period, which is $q_{t} K_{t+1}=\frac{1}{\phi} I_{t}$, as shown in equation (7). Therefore,

$$
G D P_{t}=C_{t}+\frac{1}{\phi} I_{t}=Y_{t}+\frac{1-\phi}{\phi} I_{t}
$$

where the second equality in equation (13) follows from equation (3). ${ }^{5}$

The consumption goods technology and the capital adjustment technology are interpreted as components of a two-sector model in Figure 1, which displays $K_{t+1}$ on the horizontal axis and $C_{t}$ on the vertical axis, for the case in which $0<\phi<1 .^{6}$ The two-sector production possibilities frontier is represented by the downward-sloping concave curve in Figure 1. The equilibrium combination of $C_{t}$ and $K_{t+1}$ is shown by point $E$ on the production

\footnotetext{
${ }^{5}$ In a standard neoclassical growth model with $\phi=1$, equation (13) shows that $G D P_{t}=$ $C_{t}+I_{t}=Y_{t}$. In the absence of convex adjustment costs, $I_{t}$ represents both the input to the capital adjustment technology and the value of the output of this technology. However, in the presence of convex adjustment costs $(0<\phi<1), G D P$ exceeds $C_{t}+I_{t}=Y_{t}$ because $I_{t}$ measures the input to the capital adjustment technology, not the value of the output of this technology, and the capital adjustment technology contributes value added.

${ }^{6}$ See Appendix A for a formal interpretation as a two-sector model.
} 


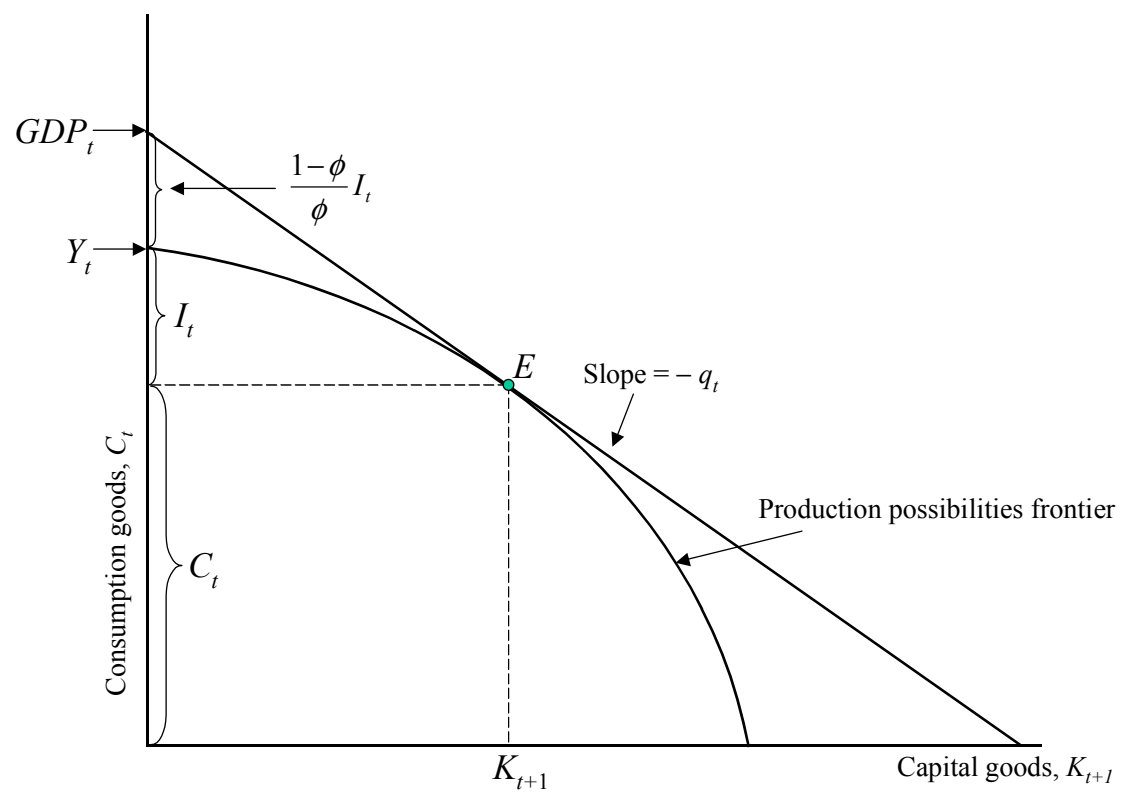

Figure 1: Production Possibilities Frontier Relating $C_{t}$ and $K_{t+1}$

possibilities frontier. The (absolute value of the) slope of the production possibilities frontier at point $E$ is the price of capital in terms of consumption, which is $q_{t}$. The value of $G D P_{t}$, measured in terms of consumption goods, is shown by the vertical intercept of the straight line through point $E$ with slope $-q_{t}$. $G D P_{t}$ exceeds $Y_{t}$ by $\frac{1-\phi}{\phi} I_{t}$, which is the value added by the capital adjustment techology. ${ }^{7}$

\footnotetext{
${ }^{7}$ The capital adjustment technology converts $I_{t}$ units of the consumption good into capital worth $q_{t} K_{t+1}$, so the value added by this technology is $q_{t} K_{t+1}-I_{t}=\frac{1-\phi}{\phi} I_{t}$. Equivalently, the value added by the capital adjustment technology equals $\nu_{t}^{K} K_{t}$, which, according to equation (10) equals $\frac{1-\phi}{\phi} I_{t}$.
} 


\section{The Social Security System}

I will specify a Social Security system in which the funding of retirement benefits can be on a pay-as-you-go basis, a fully-funded basis, or more generally, some combination of the two. Retirement benefits are determined as a hybrid of a defined-contribution system and a defined-benefit system.

Each young consumer in period $t$ pays a Social Security tax of $\tau_{t} w_{t}$. When these consumers are old in period $t+1$, they each receive a Social Security benefit of $R_{t+1} \theta_{t} w_{t} .{ }^{8}$ A defined-contribution Social Security system, in which consumers' Social Security taxes are invested on their behalf in capital, can be represented by $\theta_{t}=\tau_{t}$. In a defined-benefit system, the parameter $\theta_{t}$ can be interpreted as the replacement rate. From the viewpoint of period $t$, the present value of the Social Security benefit to be received in period $t+1$ is $\theta_{t} w_{t}$. Therefore, Social Security replaces a fraction $\theta_{t}$ of the wage income $w_{t}$, in present value. The assumption that the present value of the Social Security benefit is known when the consumer is young greatly simplifies the consumer's intertemporal consumption decision in Section 3.

The Social Security system can own a trust fund that invests in capital. Let $K_{t}^{S}$ be the amount of capital held by the Social Security system at the beginning of period $t$. Let $0 \leq \sigma_{t} \leq 1$ be the fraction of the aggregate capital stock that is held in the trust fund of the Social Security system so that

$$
K_{t}^{S}=\sigma_{t} K_{t}
$$

Using the fact that aggregate wage income earned by young consumers in period $t$ is $(1-\alpha) Y_{t}$, the sources and uses of funds for the Social Security system are given by

$$
\tau_{t}(1-\alpha) Y_{t}+R_{t} q_{t-1} \sigma_{t} K_{t}=R_{t} \theta_{t-1}(1-\alpha) Y_{t-1}+q_{t} \sigma_{t+1} K_{t+1} .
$$

The left-hand-side of equation (15) shows the sources of funds for the Social Security system in period $t$, which consist of Social Security taxes plus the value of the trust fund, including capital income, at the beginning of period $t$. The right hand side of equation (15) shows the uses of funds by the Social Security system in period $t$, which consist of retirement benefits paid to old consumers and the purchase of capital to hold in the trust fund.

\footnotetext{
${ }^{8} \mathrm{I}$ assume that $1-\tau_{t}+\theta_{t}>0$ so that the present value of lifetime income of a consumer born at the beginning of period $t,\left(1-\tau_{t}+\theta_{t}\right) w_{t}$, is positive.
} 


\section{Consumers}

At the beginning of period $t$, a continuum of consumers with measure $N_{t}$ is born. The measure of young consumers, $N_{t}$, follows a geometric random walk

$$
\ln N_{t}=\ln N_{t-1}+\varepsilon_{N, t}
$$

where the birth rate $\varepsilon_{N, t}$ is an i.i.d. random variable that is independent of $\varepsilon_{A, t}$ at all leads and lags. A high realization of $\varepsilon_{N, t}$ represents a baby boom.

Young consumers in period $t$ inelastically supply one unit of labor and earn the competitive wage rate $w_{t}$ in equation (8). A young consumer in period $t$ uses after-tax wage income $\left(1-\tau_{t}\right) w_{t}$ to consume $c_{t}^{y}$ and to purchase capital. Let $k_{t+1}^{P}$ be the amount of capital that a young consumer buys at a price $q_{t}$ per unit at the end of period $t$ to carry into period $t+1$. (The superscript $P$ denotes privately held capital to distinguish it from capital held by the Social Security system.) The purchases of consumption and capital by a young consumer satisfy

$$
q_{t} k_{t+1}^{P}=\left(1-\tau_{t}\right) w_{t}-c_{t}^{y}
$$

I assume that consumers do not have bequest motives, ${ }^{9}$ and thus they consume all available resources when they are old. Let $c_{t+1}^{o}$ be the consumption of an old consumer in period $t+1$. This consumption is financed with capital held by the consumer as well as the Social Security benefit so that

$$
c_{t+1}^{o}=R_{t+1}\left[q_{t} k_{t+1}^{P}+\theta_{t} w_{t}\right] .
$$

I assume that consumers have logarithmic utility, so the utility function of a consumer born at the beginning of period $t$ is

$$
U_{t} \equiv \ln c_{t}^{y}+\beta \ln E_{t}\left\{c_{t+1}^{o}\right\}, \quad 0<\beta<1 .
$$

I chose this utility function so that, along with the assumption that Social Security benefits equal $R_{t+1} \theta_{t} w_{t}$, the optimal consumption when young is a constant fraction of the present value of lifetime resources, which consist of after-tax wage income, $\left(1-\tau_{t}\right) w_{t}$, plus the present value of the future Social

\footnotetext{
${ }^{9}$ For an analysis of the effect of a bequest motive on the dynamic behavior of the equilibrium price of capital in the absence of Social Security, see Abel (2001).
} 
Security benefit, $\theta_{t} w_{t}$. Specifically, the optimal consumption of a young consumer in period $t$ is ${ }^{10}$

$$
c_{t}^{y}=\left(1-\tau_{t}+\theta_{t}\right) \frac{w_{t}}{1+\beta}
$$

The aggregate consumption of the young cohort, $C_{t}^{y} \equiv N_{t} c_{t}^{y}$, is calculated from equation (20) using the fact from equation (8) that $N_{t} w_{t}=(1-\alpha) Y_{t}$ to obtain

$$
C_{t}^{y}=\frac{1}{1+\beta}\left(1-\tau_{t}+\theta_{t}\right)(1-\alpha) Y_{t}
$$

\section{Equilibrium}

In this section I derive the equilibrium values of the price and quantity of capital. The solution of the equilibrium exploits four simplifying assumptions made in previous sections: (1) the production function for consumption goods is Cobb-Douglas; (2) the capital adjustment technology is log-linear; (3) consumers have logarithmic preferences; and (4) the Social Security benefit received by old consumers in period $t+1$ is $R_{t+1} \theta_{t} w_{t}$, so that a young consumer in period $t$ knows the present value of these benefits. Assumptions (3) and (4) simplify the individual consumption/saving problem, and more importantly, imply that private saving is independent of the real rate of return on saving. Without this independence, the link between private saving and the rate of return would run in both directions: private saving affects capital accumulation which affects the marginal product of capital and the rate of return; and the rate of return would affect the optimal saving of individual consumers. By severing the link in which the rate of return affects capital accumulation, it is very simple to compute the equilibrium in the economy. When assumptions (3) and (4) are combined with the loglinearity assumptions in equations (1) and (2), in the long run, the logarithm of the price of capital and the logarithm of the ratio of capital to augmented labor (defined in this section) evolve as $\mathrm{AR}(1)$ processes. The cost of this simplicity is that the model cannot capture the response of private saving to changes in interest rates, and hence would not be suited to study the effects

\footnotetext{
${ }^{10}$ Substituting equation (17) into equation (18) yields $c_{t+1}^{o}=$ $R_{t+1}\left[\left(1-\tau_{t}+\theta_{t}\right) w_{t}-c_{t}^{y}\right]$. Substituting this expression for $c_{t+1}^{o}$ into equation (19) yields $U_{t}=\ln c_{t}^{y}+\beta \ln E_{t}\left\{R_{t+1}\right\}+\beta \ln \left[\left(1-\tau_{t}+\theta_{t}\right) w_{t}-c_{t}^{y}\right]$. Differentiating this expression with respect to $c_{t}^{y}$ and setting the derivative equal to zero yields equation (20).
} 
of policies aimed at stimulating saving by increasing the (after-tax) rate of return to saving.

To derive equilibrium behavior, I begin with the observation that the aggregate consumption of the young equals the aggregate after-tax wage income of the young minus their expenditure on capital, so

$$
C_{t}^{y}=\left(1-\tau_{t}\right)(1-\alpha) Y_{t}-q_{t} K_{t+1}^{P}
$$

where $K_{t+1}^{P} \equiv N_{t} k_{t+1}^{P}$ is the total amount of capital purchased directly by young consumers at the end of period $t$. Now set the right hand side of equation (22) equal to the right hand side of equation (21) and rearrange to yield

$$
q_{t} K_{t+1}^{P}=\frac{1-\alpha}{1+\beta}\left[\left(1-\tau_{t}\right) \beta-\theta_{t}\right] Y_{t}
$$

Equation (23) shows the aggregate capital stock held directly by young consumers. To calculate the national capital stock, recall from equation (14) that the Social Security system holds capital in the amount $K_{t+1}^{S}=\sigma_{t+1} K_{t+1}$, which implies $K_{t+1}^{P}=\left(1-\sigma_{t+1}\right) K_{t+1}$. Therefore, equation (23) implies

$$
q_{t} K_{t+1}=\frac{1}{1-\sigma_{t+1}} \frac{1-\alpha}{1+\beta}\left[\left(1-\tau_{t}\right) \beta-\theta_{t}\right] Y_{t}
$$

Now use the fact from equation (7) that $q_{t} K_{t+1}=\frac{1}{\phi} I_{t}$ and the definition of the investment-output ratio $\psi_{t}$,

$$
\psi_{t} \equiv \frac{I_{t}}{Y_{t}}
$$

to rewrite equation (24) as

$$
\psi_{t}=\frac{\phi}{1-\sigma_{t+1}} \frac{1-\alpha}{1+\beta}\left[\left(1-\tau_{t}\right) \beta-\theta_{t}\right] .
$$

The sources and uses of funds for the Social Security system in equation (15) can be rewritten, using the definition of $\psi_{t}$ in equation (25) along with equations (7) and (12), as

$$
\tau_{t}+\sigma_{t} \frac{\alpha-\psi_{t}}{1-\alpha}+\left(\sigma_{t}-\sigma_{t+1}\right) \frac{1}{\phi(1-\alpha)} \psi_{t}=\frac{\theta_{t-1}}{\psi_{t-1}}\left(\alpha \phi+(1-\phi) \psi_{t}\right)
$$


Equations (26) and (27) are two equations in four variables that are determined in period $t$ : the endogenous investment-output ratio $\psi_{t}$, and the three Social Security parameters $\tau_{t}, \theta_{t}$, and $\sigma_{t+1}$. Two of the three Social Security parameters are chosen exogenously, and equations (26) and (27) can then be solved for $\psi_{t}$ and the remaining Social Security parameter.

In addition to the two endogenous variables determined by equations (26) and (27), the capital stock is an endogenous variable that evolves over time. It is convenient to focus on $\widetilde{k}_{t}$, the ratio of the capital stock to the augmented amount of labor, which is defined as ${ }^{11}$

$$
\widetilde{k}_{t} \equiv \frac{K_{t}}{A_{t}^{\frac{1}{1-\alpha}} N_{t}} .
$$

The logarithm of $\widetilde{k}_{t}$ evolves according to ${ }^{12}$

$$
\ln \widetilde{k}_{t+1}=\phi \ln \psi_{t}+[1-(1-\alpha) \phi] \ln \widetilde{k}_{t}-\frac{1}{1-\alpha} \varepsilon_{A, t+1}-\varepsilon_{N, t+1}+\ln a .
$$

The dynamic behavior of the economy is described by three equations: equations (26) and (27), which describe the dynamic behavior of $\psi_{t}$ and of the Social Security system, and equation (29) which governs the evolution of $\widetilde{k}_{t+1}$. This 3-equation system exhibits a strong form of separability. Specifically, equations (26) and (27) form an independent deterministic system that does not depend on $\widetilde{k}_{t+1}$ or on any of the shocks in equation (29).

The behavior of the independent deterministic system in equations (26) and (27) depends on the specification of Social Security policy, and I will discuss different classes of Social Security policies in Sections 7 and 8. In

\footnotetext{
${ }^{11}$ To interpret the denominator in equation (28) as the augmented amount of labor, note that the consumption goods technology in equation (1) can be rewritten as $Y_{t}=$ $K_{t}^{\alpha}\left(A_{t}^{\frac{1}{1-\alpha}} N_{t}\right)^{1-\alpha}$, where $A_{t}^{\frac{1}{1-\alpha}} N_{t}$ is the "augmented" amount of labor.

${ }^{12}$ The definition of $\psi_{t}$ in equation (25) implies that $\frac{I_{t}}{K_{t}}=\psi_{t} \frac{Y_{t}}{K_{t}}$. Therefore, the consumption goods technology in equation (1) implies $\frac{I_{t}}{K_{t}}=\psi_{t} A_{t}\left(\frac{K_{t}}{N_{t}}\right)^{\alpha-1}$. Substituting this expression for $\frac{I_{t}}{K_{t}}$ into the capital adjustment technology in equation (5) yields $K_{t+1}=a K_{t} \psi_{t}^{\phi} A_{t}^{\phi}\left(\frac{K_{t}}{N_{t}}\right)^{(\alpha-1) \phi} \cdot$ Divide both sides of this equation by $A_{t+1}^{\frac{1}{1-\alpha}} N_{t+1}$, use the definition of $\widetilde{k}_{t+1}$ in equation (28), and then take logarithms of both sides to obtain equation (29), where $\varepsilon_{A, t+1}$ is (the logarithm of) the growth rate of $T F P$ in equation (2) and $\varepsilon_{N, t+1}$ is (the logarithm of) the birth rate defined in equation (16).
} 
both of these classes of policies, there is a constant value of $\psi_{t}$ that satisfies the independent deterministic system in equations (26) and (27) and has the property that once this value is attained, $\psi_{t}$ remains constant even though stochastic shocks in equation (29) generate stochastic movements in $\widetilde{k}_{t+1}$. I will use the term "long run" to apply to situations in which $\psi_{t}$ is constant, and in Sections 7 and 8, I will examine the stability of these stationary values of $\psi_{t}$.

Equation (29) implies the following proposition.

Proposition 1 In the long run with constant $\psi_{t}, \ln \widetilde{k}_{t}$ is a stationary $A R(1)$ process if $\phi>0$, and is a random walk if $\phi=0$.

Proposition 1 implies that $\ln \widetilde{k}_{t}$ is mean-reverting if $\phi>0$, but it is not mean-reverting in the Lucas (1978) fruit-tree model, which has $\phi=0$. This mean reversion with $\phi>0$ induces mean reversion in the price of capital, $q_{t}$. The logarithm of the price of capital evolves according to ${ }^{13}$

$$
\begin{aligned}
\ln q_{t}= & {[1-(1-\alpha) \phi] \ln q_{t-1}+(1-\phi) \ln \frac{\psi_{t}}{\psi_{t-1}}-(1-\alpha) \phi \ln \phi } \\
& -(1-\alpha) \ln a+(1-\phi)\left[\varepsilon_{A, t}+(1-\alpha) \varepsilon_{N, t}\right]
\end{aligned}
$$

If $\phi=1$, the price of capital is constant and equal to $\frac{1}{a}$. For the more interesting case in which $\phi<1$, equation (30) implies the following proposition and corollaries.

Proposition 2 If $\phi<1$, the price of capital, $q_{t}$, is an increasing function of the TFP shock, $\varepsilon_{A, t}$, and of the shock to the birth rate, $\varepsilon_{N, t}$.

Corollary 1 If $0<\phi<1$, the price of capital, $\ln q_{t}$, is mean-reverting in response to TFP shocks $\varepsilon_{A, t}$ and birth rate shocks $\varepsilon_{N, t}$.

\footnotetext{
${ }^{13}$ Use the definition of $\psi_{t}$ in equation (25), the definition of $\widetilde{k}_{t}$ in equation (28), and the consumption goods technology in equation (1) to obtain

$$
\frac{I_{t}}{K_{t}}=\psi_{t} \widetilde{k}_{t}^{\alpha-1}
$$

Substitute equation (*) into equation (6) and take logarithms of both sides to obtain

$$
\ln q_{t}=-\ln a \phi+(1-\phi) \ln \psi_{t}-(1-\phi)(1-\alpha) \ln \widetilde{k}_{t} .
$$

Substitute equation (29) into equation (**), and simplify, to obtain equation (30).
} 
Corollary 2 If $\phi=0$, then the price of capital, $q_{t}$, follows a geometric random walk.

Proposition 2 and its corollaries state that the price of capital rises in response to a baby boom and rises in response to a positive shock to total factor productivity. The corollaries state that whether the price of capital reverts toward its mean in the period following the shock depends on whether capital can be accumulated. Specifically, if capital can be accumulated $(\phi>0)$, then the price of capital is mean-reverting. However, if the capital stock is fixed over time $(\phi=0)$, as in the fruit-tree model, the price of capital is not mean-reverting.

Aggregate saving is the economic channel through which a baby boom or a productivity shock affects the price of capital. A baby boom in period $t$ (i.e., a high value of $\varepsilon_{N, t}$ )-or a productivity boom in the consumption goods technology (i.e., a high value of $\varepsilon_{A, t}$ ) -causes a high value of aggregate wage income relative to the capital stock, $K_{t}$. The high wage income accrues to young consumers, who are the private savers in the economy, and thus leads to a high level of saving relative to $K_{t}$. Since aggregate investment equals aggregate saving in a closed economy ${ }_{1}{ }^{14}$ aggregate investment is high relative to $K_{t}$. The high value of aggregate investment relative to the capital stock drives up the price of capital along the upward-sloping supply curve of capital in equation (6), provided that $\phi<1$. As a result of the high value of investment in period $t$, the capital stock in the following period, $K_{t+1}$, is high, provided that $\phi>0$. The high value of $K_{t+1}$ reduces the price of capital at any level of investment in period $t+1$, thus putting downward pressure on the price of capital and generating mean reversion.

Equation (30) and the fact that $\psi_{t}$ is constant in the long run when the Social Security parameters are constant imply the following proposition. ${ }^{15}$

Proposition 3 In the long run, the price of capital, $q_{t}$, is independent of

\footnotetext{
${ }^{14}$ More precisely, $I_{t}=\phi q_{t} K_{t+1}=\frac{\phi}{1-\sigma_{t+1}} q_{t} K_{t+1}^{P}$, where the first equality follows from equation (7), the second equality follows from equation (14), and $q_{t} K_{t+1}^{P}$ is the aggregate saving of young consumers in period $t$.

${ }^{15}$ If Social Security were to have an effect on the price of capital in the long run, the channel would be through the investment-capital ratio $\frac{I_{t}}{K_{t}}$ (See equation (6)). However, in the long run, the investment-output ratio $\psi_{t}$ is constant, so equation $\left(^{*}\right)$ in footnote 13 implies that $\frac{I_{t}}{K_{t}}=\psi \widetilde{k}_{t}^{\alpha-1}$. Although Social Security can potentially affect the long-run value of $\psi$ (see Section 7), I will show in footnote 17 that the elasticity of $\widetilde{k}_{t}$ with respect to $\psi$ is $\frac{1}{1-\alpha}$. Therefore, the product $\psi \widetilde{k}_{t}^{\alpha-1}=\frac{I_{t}}{K_{t}}$ is unaffected by changes in $\psi$.
} 
the investment-output ratio $\psi$ and the constant values of the Social Security parameters $\tau_{t}, \theta_{t}$, and $\sigma_{t}$.

Recall from equation (7) that the market value of the capital stock, $q_{t} K_{t+1}$, is $\frac{1}{\phi} I_{t}$, and observe that equation (13) states that $G D P_{t}=Y_{t}+\frac{1-\phi}{\phi} I_{t}$. The ratio of the market value of the capital stock to $G D P$ is given by the following proposition.

Proposition $4 \frac{q_{t} K_{t+1}}{G D P_{t}}=\frac{\psi_{t}}{\phi+(1-\phi) \psi_{t}}$.

The ratio $\frac{q_{t} K_{t+1}}{G D P_{t}}$ is independent of the stochastic shocks in the model and is independent of $\widetilde{k}_{t}$. Indeed, in the long run $\psi_{t}$ remains constant, and thus $\frac{q_{t} K_{t+1}}{G D P_{t}}$ is constant despite the presence of stochastic shocks in the long run. The huge increase in the value of stocks in the 1990s would seem to indicate that $\frac{q_{t} K_{t+1}}{G D P_{t}}$ increased sharply during this period. However, conventionally measured GDP may understate $G D P$ when there is a stock market boom and high capital accumulation, so the ratio $\frac{q_{t} K_{t+1}}{G D P_{t}}$ may, in fact, have increased far less than would appear from conventional data. ${ }^{16}$

\section{$5 \quad$ Laissez Faire}

Henceforth, we will assume that $\phi>0$, so that economy can accumulate capital and the capital stock is determined endogenously. In this section, we examine capital accumulation under laissez-faire. Under laissez faire, there is no Social Security system. Formally, $\tau_{t}=\theta_{t}=\sigma_{t}=0$ for all $t$. Equation (26) immediately implies the following proposition.

Proposition 5 Under laissez faire $\left(\tau_{t}=\theta_{t}=\sigma_{t}=0\right.$ for all $\left.t\right), \psi_{t}=\psi_{L F} \equiv$ $\phi \frac{\beta}{1+\beta}(1-\alpha)$ for all $t$.

Under laissez faire, national saving equals private saving, which equals the saving of the young generation. The aggregate income of young consumers is a constant share $1-\alpha$ of output $Y_{t}$. They save a constant fraction $\frac{\beta}{1+\beta}$ of their income, so the saving of young consumers is $\frac{\beta}{1+\beta}(1-\alpha) Y_{t}$. Equivalently, the value of capital purchased by young consumers, $q_{t} K_{t+1}$, equals $\frac{\beta}{1+\beta}(1-\alpha) Y_{t} . \quad$ Since $I_{t}=\phi q_{t} K_{t+1}$ (equation $\left.(7)\right), I_{t}=\phi \frac{\beta}{1+\beta}(1-\alpha) Y_{t}$, which implies Proposition 5.

\footnotetext{
${ }^{16}$ See Hall (2001) for a discussion of this point.
} 


\section{The Golden Rule and Dynamic Efficiency}

In the long run with constant values of the Social Security parameters, the value of $\psi_{t}$ is constant. The Golden Rule value of $\psi$, denoted $\psi_{G R}$, is the constant value of $\psi_{t}$ that maximizes the long-run value of $\widetilde{c}_{t} \equiv \frac{C_{t}}{A_{t}^{1-\alpha} N_{t}}$, the amount of consumption per augmented unit of labor. To derive an expression for $\widetilde{c}_{t}$, use the definition of $\psi_{t}$ from equation (25) and the consumption goods technology in equation (1) to rewrite equation (3) as

$$
C_{t}=\left(1-\psi_{t}\right) A_{t} K_{t}^{\alpha} N_{t}^{1-\alpha} .
$$

Divide both sides of equation (31) by $A_{t}^{\frac{1}{1-\alpha}} N_{t}$, use the definitions $\widetilde{c}_{t} \equiv \frac{C_{t}}{A_{t}^{\frac{1}{1-\alpha}} N_{t}}$ and $\widetilde{k}_{t} \equiv \frac{K_{t}}{A_{t}^{\frac{1}{1-\alpha}} N_{t}}$, and use the fact that $\psi_{t}$ is constant in the long run to obtain

$$
\widetilde{c}_{t}=(1-\psi) \widetilde{k}_{t}^{\alpha}
$$

To determine the value of $\psi$ that maximizes consumption per augmented unit of labor in the long run, differentiate equation (32) with respect to $\psi$ to obtain

$$
\frac{\partial \widetilde{c}_{t}}{\partial \psi}=\left(\alpha \frac{1-\psi}{\psi} \frac{\partial \ln \widetilde{k}_{t}}{\partial \ln \psi}-1\right) k_{t}^{\alpha} .
$$

Setting the derivative $\frac{\partial \widetilde{c}_{t}}{\partial \psi}$ in equation (33) equal to zero and $\operatorname{using}^{17}$ $\frac{\partial \ln \widetilde{k}_{t}}{\partial \ln \psi}=\frac{1}{1-\alpha}$ yields the following proposition.

Proposition 6 The Golden Rule, which maximizes consumption per augmented unit of labor in the long run, is attained by $\psi=\psi_{G R} \equiv \alpha$.

This proposition can be interpreted using the results on dynamic efficiency under uncertainty in Abel, Mankiw, Summers, and Zeckhauser (1989), hereafter AMSZ. Proposition 2 in AMSZ states that if, in every period, the rate of return on risky capital exceeds the growth rate of the value of the capital

\footnotetext{
${ }^{17}$ Rewrite the expression for $\ln \widetilde{k}_{t}$ in equation (29) as $\ln \widetilde{k}_{t}=\frac{1}{1-\alpha}\left(\ln \psi+\frac{1}{\phi} \ln a\right)+$ $\sum_{j=0}^{\infty}[1-(1-\alpha) \phi]^{j}\left[-\frac{1}{1-\alpha} \varepsilon_{A, t-j}-\varepsilon_{N, t-j}\right]$. Inspection of this equation reveals that the desired elasticity, $\frac{\partial \ln \widetilde{k}_{t}}{\partial \ln \psi}$, is $\frac{1}{1-\alpha}$.
} 
stock, the economy is dynamically efficient. If, in every period, the rate of return on capital is less than the growth rate of the value of the capital stock, the economy has a dynamically inefficient overaccumulation of capital.

In AMSZ the price of capital is fixed at unity, but here the price of capital varies endogenously. With a variable price of capital, the gross growth rate of the value of the capital stock is $G_{t} \equiv \frac{q_{t} K_{t+1}}{q_{t-1} K_{t}}$. Define

$$
\rho_{t} \equiv \frac{R_{t}}{G_{t}}
$$

as the ratio of the rate of return on capital to the growth rate of the value of the capital stock. Extending the results of AMSZ to the case with a variable price of capital, an economy is dynamically efficient if $\rho_{t}>1$ in every period, but it is dynamically inefficient if $\rho_{t}<1$ in every period. If $\rho_{t}=1$ in every period, the economy attains the Golden Rule. ${ }^{18}$

To obtain a simple expression for $\rho_{t}$ in the long run in this model, use the expression for $R_{t}$ in equation (12), the expression for $q_{t} K_{t+1}$ in equation (7), and the fact that the investment-output ratio $\psi_{t}$ is constant in the long run to rewrite equation (34) as

$$
\rho_{t}=\rho \equiv 1+\frac{\phi}{\psi}(\alpha-\psi)
$$

Equation (35) shows that if $\phi>0$, an economy is dynamically efficient if $\psi<\alpha$. It has a dynamically inefficient overaccumulation of capital if $\psi>\alpha$, and it attains the Golden Rule if $\psi=\alpha$.

AMSZ present an alternative criterion for dynamic efficiency in their Proposition 1. Specifically, if, in every period, the contribution of capital to output is larger than the flow of resources used to create capital, the economy is dynamically efficient. In this model, the contribution of capital to the gross production of consumption goods is $\alpha Y_{t}$ and the flow of resources diverted from consumption to the creation of capital is $I_{t}$. Thus, the contribution of capital exceeds its use of resources by $\alpha Y_{t}-I_{t}=(\alpha-\psi) Y_{t}$ in period $t$. Consistent with the results discussed above, this comparison of the contribution of capital to its use of resources indicates that the economy is dynamically efficient if $\psi<\alpha$, is dynamically inefficient if $\psi>\alpha$, and attains the Golden Rule if $\psi=\alpha \cdot{ }^{19}$

\footnotetext{
${ }^{18}$ Abel (2002) shows in a deterministic framework that in the presence of convex adjustment costs, the Golden Rule is characterized by $R_{t}=G_{t}$ (which implies $\rho_{t}=1$ ).

${ }^{19}$ The AMSZ conditions for dynamic efficiency and dynamic inefficiency are sufficient
} 


\subsection{Golden Rule Social Security Policies}

Since $\psi_{t}=\psi_{t-1}=\alpha$ in an economy that has attained the Golden Rule, equation (27) implies that $\tau_{t}=\theta_{t-1}$ in the Golden Rule steady state of an economy with constant values of the Social Security parameters $\tau_{t}, \theta_{t}$, and $\sigma_{t}{ }^{20}$ Therefore, equation (26) implies the following proposition.

Proposition 7 When the Golden Rule steady state is attained in the long run with constant values of the Social Security parameters $\left(\tau_{t}=\tau, \theta_{t}=\theta\right.$, and $\left.\sigma_{t}=\sigma\right), \tau=\theta=\theta_{G R} \equiv \frac{1}{\phi} \frac{1}{1-\alpha}\left[\psi_{L F}-(1-\sigma) \alpha\right]$.

Corollary 3 In a pure pay-as-you-go system $(\sigma=0)$, when the Golden Rule steady state is attained in the long run with constant values of the Social Security parameters, $\tau=\theta=\frac{1}{\phi} \frac{1}{1-\alpha}\left[\psi_{L F}-\alpha\right]$.

Corollary 3 implies that if $\psi_{L F}<\alpha$, so the laissez-faire economy is dynamically efficient, the Golden Rule is attained by negative values of $\tau$ and $\theta$. Negative values of $\tau$ and $\theta$ imply that young consumers receive a subsidy and old consumers pay a tax. Since subsidizing the income of young consumers and increasing the taxes paid by old consumers both increase the saving of young consumers, this tax/subsidy scheme will increase national saving and investment toward the Golden Rule. On the other hand, if the laissez-faire economy is dynamically inefficient $\left(\psi_{L F}>\alpha\right)$, national saving and investment must be reduced to attain the Golden Rule. In this case, positive values of $\tau$ and $\theta$ are required to attain the Golden Rule.

\section{Social Security with Constant $\theta_{t}$ and $\sigma_{t}$}

In this section I consider the class of Social Security policies in which the replacement rate $\theta_{t}$ and the trust fund ratio $\sigma_{t}, 0 \leq \sigma_{t}<1$, are constant.

but not necessary. In particular, these conditions do not apply when $\rho_{t}$ is sometimes greater than one and sometimes less than one. Conveniently, in this model, $\rho_{t}$ is constant in the long run, so the AMSZ conditions are decisive. For situations in which $\rho_{t}$ is not always greater than or always less than one, dynamic efficiency or inefficiency could be assessed by extending the results of Zilcha (1991) to the case with convex adjustment costs and stochastic population growth.

${ }^{20}$ Two of the three Social Security parameters $\left(\tau_{t}, \theta_{t}\right.$, and $\left.\sigma_{t}\right)$ are chosen exogenously. Equation (26) implies that if any two of these three parameters are constant, and if $\psi_{t}$ is constant, then the third Social Security parameter is constant. 
This class includes $\sigma_{t} \equiv 0$, which represents a pay-as-you-go system. A constant replacement rate $\theta_{t}$ represents a defined-benefit system in which (the present value of) Social Security benefits are a constant fraction of wage income when young.

Setting $\theta_{t}=\theta$ and $\sigma_{t}=\sigma$ for all $t$, equations (26) and (27) can be rewritten as

$$
\psi_{t}=\frac{\phi}{1-\sigma} \frac{1-\alpha}{1+\beta}\left[\left(1-\tau_{t}\right) \beta-\theta\right] .
$$

and

$$
\tau_{t}+\sigma \frac{\alpha-\psi_{t}}{1-\alpha}=\frac{\theta}{\psi_{t-1}}\left(\alpha \phi+(1-\phi) \psi_{t}\right) .
$$

Equations (36) and (37) govern the evolution of $\psi_{t}$ and $\tau_{t}$. This system of two equations in $\psi_{t}$ and $\tau_{t}$ can be expressed as a single difference equation in $\psi_{t}$ by substituting equation (37) into equation (36) and rearranging to obtain

$$
\psi_{t}=h\left(\psi_{t-1}\right) \equiv \frac{((1-\alpha)(\beta-\theta)+\sigma \alpha \beta) \psi_{t-1}-\alpha \beta \phi \theta(1-\alpha)}{\left(\frac{(1-\sigma)(1+\beta)}{\phi}+\sigma \beta\right) \psi_{t-1}+\beta \theta(1-\phi)(1-\alpha)} .
$$

To find the steady-state values of $\psi_{t}$, set $\psi_{t}=\psi_{t-1}=\psi$ in equation (38) and rewrite this equation as

$$
H(\psi, \theta, \sigma)=0
$$

where

$$
\begin{aligned}
H(\psi, \theta, \sigma) \equiv & {\left[\frac{(1-\sigma)(1+\beta)}{\phi}+\sigma \beta\right] \frac{1}{1-\alpha} \psi^{2} } \\
& -\left[\beta+\sigma \frac{\alpha \beta}{1-\alpha}-\theta-(1-\phi) \beta \theta\right] \psi+\beta \theta \phi \alpha .
\end{aligned}
$$

For given values of the Social Security policy parameters $\theta$ and $\sigma$, the function $H(\psi, \theta, \sigma)$ is quadratic in $\psi$ and thus has at most two real roots. If $H(\psi, \theta, \sigma)=0$ has real roots, these roots are stationary values of the difference equation in equation (38). 


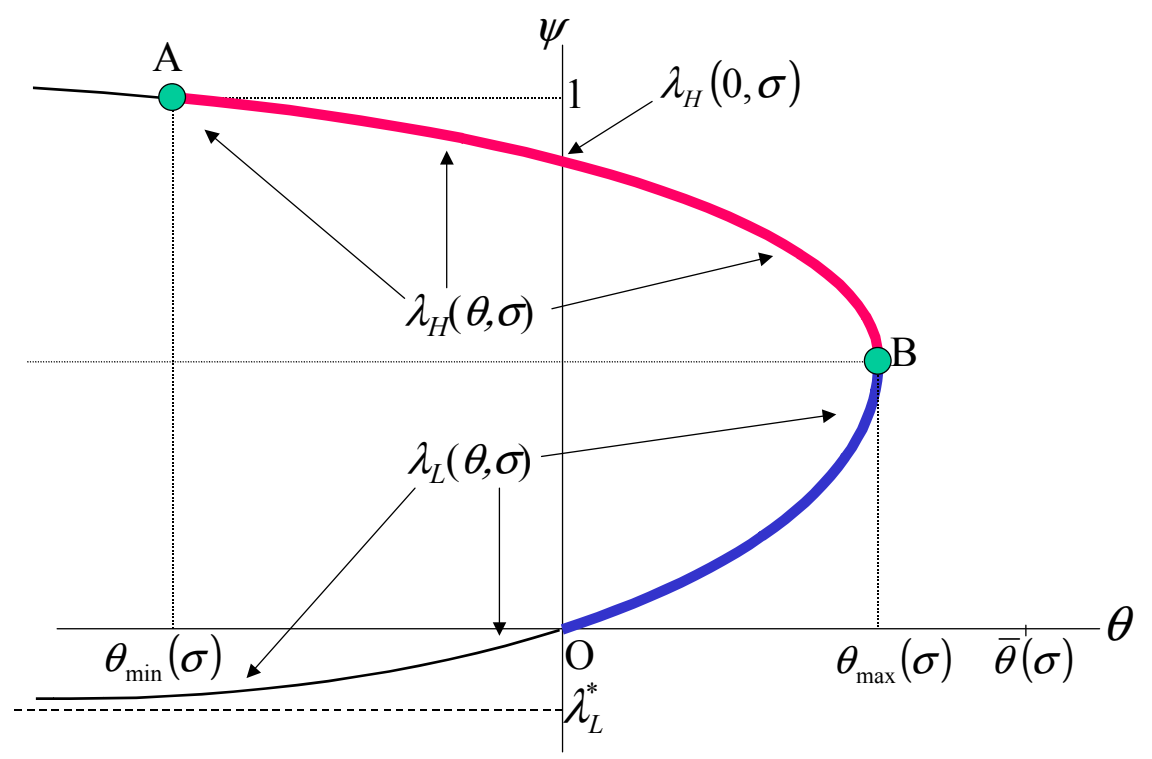

Figure 2: Roots of $H(\psi, \theta, \sigma)=0$.

Lemma 8 in Appendix B fully describes the roots of $H(\psi, \theta, \sigma)=0$, and Figure 2 shows the two real roots $\lambda_{L}(\theta, \sigma) \leq \lambda_{H}(\theta, \sigma)$ as functions of the replacement rate $\theta$ for a given value of the trust fund ratio $\sigma$. The horizontal axis measures $\theta$ and the vertical axis shows the values of $\lambda_{i}(\theta, \sigma), i=L, H$. These roots represent the steady-state values of the investment-output ratio $\psi$. For values of $\theta<\theta_{\max }(\sigma)$, there are two real roots. The economically interesting roots lie between 0 and 1 , and these roots are represented by the thick portion of the curve, which is arc OBA. For $\theta=0$, one root equals zero and the other root is positive and equal to $\lambda_{H}(0, \sigma)=\phi \frac{(1-\alpha) \beta+\sigma \alpha \beta}{(1-\sigma)(1+\beta)+\sigma \beta \phi}<1$. Under laissez faire, $\theta=0$ and $\sigma=0$, so the positive root becomes $\lambda_{H}(0,0)=$ $\phi \frac{\beta}{1+\beta}(1-\alpha) \equiv \psi_{L F}$, which is consistent with Proposition 5 .

For negative values of $\theta$, the equation $H(\psi, \theta, \sigma)=0$ has one positive root. Provided that the negative value of $\theta$ is greater than $\theta_{\min }(\sigma) \equiv-\frac{1}{\phi} \frac{1-\sigma}{1-\alpha}$, this root is less than one and thus is economically interesting. 
For positive values of $\theta$ less than $\theta_{\max }(\sigma)$, the quadratic equation $H(\psi, \theta, \sigma)=$ 0 has two positive roots less than one.

A stationary point, $\psi_{t}=\psi_{t-1}=\lambda$ of the difference equation in equation (38) is locally stable if the derivative $h^{\prime}(\lambda)$ satisfies $-1<h^{\prime}(\lambda)<1$. Lemma 9 in Appendix B, which describes the stability of a class of difference equations containing the difference equation in equation (38), implies the following proposition. ${ }^{21}$

Proposition 8 Suppose that $\theta_{\min }(\sigma)<\theta<\theta_{\max }(\sigma)$. Then $H(\psi, \theta, \sigma)=0$ has two distinct real roots $\lambda_{L}(\theta, \sigma)<\lambda_{H}(\theta, \sigma)$. The larger root, $\lambda_{H}(\theta, \sigma)$, is positive and $\psi=\lambda_{H}(\theta, \sigma)$ is a stable steady state of equation (38). If $\lambda_{L}(\theta, \sigma)>0$ (equivalently, if $\theta>0$ ), then $\psi=\lambda_{L}(\theta, \sigma)$ is an unstable steady state of equation (38).

Corollary 4 The laissez faire steady state, $\psi=\lambda_{H}(0,0)=\psi_{L F} \equiv \phi \frac{(1-\alpha) \beta}{1+\beta}$, is stable.

Proposition 8 implies that the economically interesting steady states that are stable lie along the arc $A B$ in Figure 2. This arc slopes downward and thus implies the following proposition.

Proposition 9 Suppose that $\theta_{\min }(\sigma)<\theta<\theta_{\max }(\sigma)$. In the class of Social Security policies with constant values of $\theta_{t}$ and $\sigma_{t}$, the unique stable steadystate value of $\psi$ is a decreasing function of $\theta$.

The primary effect reflected in Proposition 9 is that an increase in the replacement rate $\theta$ increases retirement income and thus induces young consumers to consume more and save less. The fall in national saving reduces the investment-output ratio $\psi$.

Corollary 6 in Appendix B implies the following proposition.

Proposition 10 Suppose that $\theta_{\min }(\sigma)<\theta<\theta_{\max }(\sigma)$. In the class of Social Security policies with constant values of $\theta_{t}$ and $\sigma_{t}$, the unique stable steady-state value of $\psi$ is an increasing function of $\sigma$.

An increase in $\sigma$ increases the saving of the Social Security system, thereby increasing national saving and thus increasing $\psi$.

\footnotetext{
${ }^{21}$ To apply Lemma 9 to equation (38), set $a=(1-\alpha)(\beta-\theta)+\sigma \alpha \beta ; b=-\alpha \beta \phi \theta(1-\alpha)$; $d=\frac{(1-\sigma)(1+\beta)}{\phi}+\sigma \beta$; and $e=\beta \theta(1-\phi)(1-\alpha)$. Note that $d>0$, be $\leq 0$, and $a+e$ $=(1-\alpha)[(1+\beta(1-\phi)) \bar{\theta}(\sigma)-(1-\beta(1-\phi)) \theta]$, where $\bar{\theta}(\sigma) \equiv \frac{\beta\left(1+\sigma \frac{\alpha}{1-\alpha}\right)}{1+\beta(1-\phi)}$. Since $\theta \leq$ $\theta_{\max }(\sigma)$ and Lemma 6 states that $\theta_{\max }(\sigma)<\bar{\theta}(\sigma), a+e>0$.
} 


\section{Defined-Contribution Social Security}

In this section I consider a defined-contribution Social Security system in which benefits paid to retirees equal the amount they would receive if their taxes were invested on their behalf in capital. However, the Social Security system I analyze need not be fully funded.

In a defined-contribution Social Security system, a young consumer with a wage income of $w_{t}$ in period $t$ pays a tax of $\tau_{t} w_{t}$ in period $t$ and receives a benefit of $R_{t+1} \tau_{t} w_{t}$ in period $t+1$, so $\theta_{t}=\tau_{t}$. I will assume that

$$
0 \leq \tau_{t}=\theta_{t}<\frac{\beta}{1+\beta}
$$

so that the consumption of young consumers is less than their disposable income. ${ }^{22}$

I will confine attention to policies with constant values of $\tau_{t}$ and $\theta_{t}$. Setting $\theta_{t}=\tau_{t}=\tau$ in equations (26) and (27) yields

$$
\psi_{t}=\frac{\phi}{1-\sigma_{t+1}} \frac{1-\alpha}{1+\beta}[(1-\tau) \beta-\tau]
$$

and

$$
\tau+\sigma_{t} \frac{\alpha-\psi_{t}}{1-\alpha}+\left(\sigma_{t}-\sigma_{t+1}\right) \frac{1}{\phi(1-\alpha)} \psi_{t}=\frac{\tau}{\psi_{t-1}}\left(\alpha \phi+(1-\phi) \psi_{t}\right)
$$

Equations (42) and (43) govern the dynamic behavior of the investmentoutput ratio $\psi_{t}$ and the trust fund ratio $\sigma_{t}$. This two-equation system can be written as a difference equation in $\psi_{t}$ by solving equation (42) for $\sigma_{t+1}$, substituting the result and an analogous result for $\sigma_{t}$ into equation (43), and rearranging to obtain

$$
\psi_{t}=\frac{(\alpha+\beta) \psi_{t-1}-\alpha \beta \phi(1-\alpha)}{(1+\beta) \psi_{t-1}+(1-\phi) \beta(1-\alpha)} .
$$

The steady-state values of $\psi_{t}$ are found by setting $\psi_{t}=\psi_{t-1}=\psi$ in equation (44) and rewriting this equation to obtain the following quadratic

\footnotetext{
${ }^{22}$ In a defined-contribution system, a young consumer has disposable income of $\left(1-\tau_{t}\right) w_{t}$ and consumes $c_{t}^{y}=\frac{1}{1+\beta} w_{t}$. Consumption will be smaller than disposable income if $\frac{1}{1+\beta}<1-\tau_{t}$, or equivalently if $\tau_{t}<\frac{\beta}{1+\beta}$.
} 
equation in $\psi$

$$
[(1+\beta) \psi-(1-\alpha) \beta \phi](\psi-\alpha)=0 .
$$

The quadratic equation in (45) has two roots: $\alpha$ and $\psi_{L F} \equiv(1-\alpha) \frac{\beta}{1+\beta} \phi$. These roots, along with Lemma 9 in Appendix B, imply the following proposition. $^{23}$

Proposition 11 If $\tau_{t}=\theta_{t}$ is constant for all $t$, the steady-state value of the investment-output ratio is either the laissez faire value, $\psi_{L F} \equiv(1-\alpha) \frac{\beta}{1+\beta} \phi$, or the Golden Rule value, $\alpha$, regardless of the constant value of $\theta_{t}=\tau_{t}$. The steady state with the higher value of $\psi$ is stable, and the steady state with the lower value of $\psi$ is unstable.

To determine the state-state value of the trust fund ratio, assume that $\psi_{t}$ and $\sigma_{t+1}$ are constant and rewrite equation (42) using the definition of $\psi_{L F}$ in Proposition 5 to obtain

$$
1-\sigma=\left(1-\frac{1+\beta}{\beta} \tau\right) \frac{\psi_{L F}}{\psi}
$$

Equation (46) implies that in the steady state with $\psi=\psi_{L F}$, the trust fund ratio is

$$
\sigma=\frac{1+\beta}{\beta} \tau
$$

With the trust fund ratio in equation (47), the value of the Social Security trust fund at the end of period $t, q_{t} K_{t+1}^{S}$, equals the value of the Social Security taxes collected from young consumers in period $t, \tau_{t}(1-\alpha) Y_{t}{ }^{24}$ Thus, taxes collected from young consumers are fully invested on their behalf by the Social Security system at the market rate of return. When these consumers are old, they receive Social Security benefits equal to the amount they would have had available from investing their Social Security taxes at the market rate of return. Therefore, the lifetime profile of optimal consumption is unchanged by the Social Security system, and the steady state is the laissez

\footnotetext{
${ }^{23}$ To apply Lemma 9 to equation (44), set $a=\alpha+\beta ; b=-\alpha \beta \phi(1-\alpha) ; d=1+\beta$; and $e=(1-\phi) \beta(1-\alpha)$, so $d>0, b e<0$, and $a+e>0$.

${ }^{24} q_{t} K_{t+1}^{S}=\sigma q_{t} K_{t+1}=\sigma \frac{1}{\phi} I_{t}=\sigma \frac{1}{\phi} \psi_{L F} Y_{t}=\sigma \frac{\beta}{1+\beta}(1-\alpha) Y_{t}=\tau(1-\alpha) Y_{t}$, where the first equality follows from the definition of the trust fund ratio $\sigma$, the second equality follows from equation (7), the third equality follows from $\psi=\frac{I_{t}}{Y_{t}}$ and the fact that $\psi=\psi_{L F}$, the fourth equality from the definition of $\psi_{L F}$, and the fifth equality follows from equation (47).
} 
faire steady state. This invariance to the presence or size of a fully-funded defined-contribution Social Security system is an example of the Ricardian Equivalence Proposition.

In the Golden Rule steady state, the trust fund ratio given by (46) is just the right size to make the national capital-labor ratio equal to the Golden Rule value. ${ }^{25}$

\section{Concluding Remarks}

I have shown that a baby boom will increase the price of capital. Specifically, when baby boomers are in the labor force earning wage income, national saving and investment are high. In the presence of convex adjustment costs, or equivalently in a two-sector economy that produces consumption goods and capital goods, a high rate of investment can be achieved only by driving up the supply price of capital. Thus, a baby boom increases the price of capital. However, the price of capital displays mean reversion (provided that $\phi>0$ ), so that this increase in the price of capital is followed by a fall in the price of capital in the following period.

I included Social Security in the paper because it is an important part of retirement income, and saving for retirement is a major component of the model. I have shown how Social Security can be used to change national saving and investment in the long run, and even to achieve the Golden Rule. Although Social Security can affect the investment-output ratio in the long run, it does not affect the price of capital in the long run. However, in the model in this paper, the Social Security trust fund is held entirely in capital. Further research would be needed to analyze any long-run impact of the Social Security system on stock prices if the trustees of the trust fund can decide how to allocate the assets between risky capital and riskless bonds.

\footnotetext{
${ }^{25}$ In a Golden Rule steady state, $q_{t} K_{t+1}=\frac{1}{\phi} I_{t}=\frac{\alpha}{\phi} Y_{t}$. With a defined-contribution social security system, the value of the capital held directly by young consumers at the end of period $t$ equals disposable income, $(1-\tau)(1-\alpha) Y_{t}$, minus their consumption, which is $\frac{1}{1+\beta}(1-\alpha) Y_{t}$. Thus, $q_{t} K_{t+1}^{P}=(1-\tau)(1-\alpha) Y_{t}-\frac{1}{1+\beta}(1-\alpha) Y_{t}=$ $\left(1-\tau \frac{1+\beta}{\beta}\right) \frac{\beta}{1+\beta}(1-\alpha) Y_{t}$. Therefore, $1-\sigma=\frac{q_{t} K_{t+1}^{P}}{q_{t} K_{t+1}}=\left(1-\tau \frac{1+\beta}{\beta}\right) \frac{\psi_{L F}}{\alpha}$, which satisfies equation (46) with $\psi=\alpha$.
} 


\section{A Interpretation as a Two-Sector Model}

This appendix interprets the production and capital adjustment technologies in equations (1) and (5) as a two-sector model in which labor, $N_{t}$, and capital, $K_{t}$, are combined in period $t$ to produce aggregate consumption, $C_{t}$, in period $t$ and a capital stock, $K_{t+1}$, in period $t+1$. Since $I_{t}$ is the gross production of consumption goods not consumed in period $t$,

$$
I_{t}=A_{t} K_{t}^{\alpha} N_{t}^{1-\alpha}-C_{t} .
$$

Substitute equation (A.1) into equation (5) and rearrange to obtain the following representation of the two-sector technology

$$
C_{t}=A_{t} K_{t}^{\alpha} N_{t}^{1-\alpha}-a^{-\frac{1}{\phi}} K_{t+1}^{\frac{1}{\phi}} K_{t}^{1-\frac{1}{\phi}} .
$$

Given $N_{t}$ and $K_{t}$, equation (A.2) shows that $C_{t}$ is a decreasing, concave function of $K_{t+1}$, so that the production possibilities frontier between $C_{t}$ and $K_{t+1}$ slopes downward and is concave to the origin (see Figure 1 in Section 1 ). The right hand side of equation (A.2) is a linearly homogeneous function of $N_{t}, K_{t}$, and $K_{t+1}$. Thus, a doubling of the factor inputs $N_{t}$ and $K_{t}$ in period $t$ makes possible the doubling of the outputs $C_{t}$ and $K_{t+1}$.

Differentiating equation (A.2) with respect to $K_{t+1}$ and using equation (5) yields

$$
\frac{\partial C_{t}}{\partial K_{t+1}}=-\frac{1}{\phi a}\left(\frac{I_{t}}{K_{t}}\right)^{1-\phi} .
$$

Comparing equations (6) and (A.3) verifies that price of capital $q_{t}$ equals $-\frac{\partial C_{t}}{\partial K_{t+1}}$.

The rental earned by capital in period $t$, expressed in units of consumption, can be calculated by differentiating equation (A.2) with respect to $K_{t}$ and using equations (1) and (5) to obtain

$$
\frac{\partial C_{t}}{\partial K_{t}}=\alpha \frac{Y_{t}}{K_{t}}+\frac{1-\phi}{\phi} \frac{I_{t}}{K_{t}} .
$$

Inspection of equations (9), (10), and (A.4) verifies that marginal product of capital, measured in terms of consumption goods, equals $\nu_{t}^{C}+\nu_{t}^{K}$. 


\section{B Equilibrium Values of $\psi$ and the Social Se- curity Parameters}

This Appendix proves lemmas and corollaries that are useful in characterizing the equilibrium values of $\psi$ and the Social Security parameters.

Lemma 1 Suppose that $0<\phi \leq 1$. Define $\bar{\theta}(\sigma) \equiv \frac{\beta\left(1+\sigma \frac{\alpha}{1-\alpha}\right)}{1+\beta-\beta \phi}>0, \lambda_{L}^{*} \equiv$ $-\frac{\beta \phi \alpha}{1+(1-\phi) \beta}<0$, and $0<\psi_{0}(\sigma) \equiv \phi \frac{(1-\alpha) \beta+\sigma \alpha \beta}{(1-\sigma)(1+\beta)+\sigma \beta \phi}<1 .^{26} H(\psi, \theta, \sigma)$, defined in equation (40), has the following properties:

(1) $\operatorname{sign}(H(0, \theta, \sigma))=\operatorname{sign}(\theta)$.

(2) $\operatorname{sign}\left(H_{\psi}(0, \theta, \sigma)\right)=\operatorname{sign}(\theta-\bar{\theta}(\sigma))$.

(3) $\operatorname{sign}\left(H_{\theta}(\psi, \theta, \sigma)\right)=\operatorname{sign}\left(\psi-\lambda_{L}^{*}\right)$.

(4) $\operatorname{sign}\left(H_{\sigma}(\psi, \theta, \sigma)\right)=-\operatorname{sign}\left(\psi\left(\psi-\lambda_{L}^{*}\right)\right)$

(5) $H\left(\psi_{0}(\sigma), 0, \sigma\right)=0$.

Proof. (1) $H(0, \theta, \sigma)=\beta \theta \phi \alpha$ and $\beta \phi \alpha>0$. (2) $H_{\psi}(0, \theta, \sigma)=$ $[1+(1-\phi) \beta](\theta-\bar{\theta}(\sigma))$ and $1+(1-\phi) \beta \geq 0$. (3) $H_{\theta}(\psi, \theta, \sigma)=[1+(1-\phi) \beta]\left(\psi-\lambda_{L}^{*}\right)$ and $1+(1-\phi) \beta>0$. (4) $H_{\sigma}(\psi, \theta, \sigma)=-\frac{1}{1-\alpha} \frac{1}{\phi}[1+(1-\phi) \beta] \psi\left(\psi-\lambda_{L}^{*}\right)$ and $1+(1-\phi) \beta \geq 0$. (5) $H(\psi, 0, \sigma)=\left[\left(\frac{(1-\sigma)(1+\beta)}{\phi}+\sigma \beta\right) \frac{1}{1-\alpha} \psi-\left(\beta+\sigma \frac{\alpha \beta}{1-\alpha}\right)\right] \psi$. Substituting $\psi_{0}$ for $\psi$ yields $H\left(\psi_{0}, 0, \sigma\right)=0$.

Lemma 2 Define $\psi_{m}(\theta, \sigma) \equiv \arg \min _{\psi} H(\psi, \theta, \sigma)$. Then sign $\left(\psi_{m}(\theta, \sigma)\right)=$ $-\operatorname{sign}(\theta-\bar{\theta}(\sigma))$ and $\frac{\partial \psi_{m}(\theta, \sigma)}{\partial \theta}<0$. If $\theta<\bar{\theta}(\sigma)$, then $\frac{\partial \psi_{m}(\theta, \sigma)}{\partial \sigma}>0$.

Proof. Since $H_{\psi \psi}(\psi, \theta, \sigma)=2\left[\frac{(1-\sigma)(1+\beta)}{\phi}+\sigma \beta\right] \frac{1}{1-\alpha}>0, \operatorname{sign}\left(\psi_{m}(\theta, \sigma)\right)=$ $-\operatorname{sign}\left(H_{\psi}(0, \theta, \sigma)\right)=-\operatorname{sign}(\theta-\bar{\theta}(\sigma))$ where the final equality follows from statement (2) of Lemma 1.

Note that $\psi_{m}(\theta, \sigma)$ satisfies $H_{\psi}\left(\psi_{m}(\theta, \sigma), \theta, \sigma\right)=0$. Differentiating this expression with respect to $\theta$ yields $H_{\psi \psi}\left(\psi_{m}(\theta, \sigma), \theta, \sigma\right) \frac{\partial \psi_{m}(\theta, \sigma)}{\partial \theta}+H_{\psi \theta}\left(\psi_{m}(\theta, \sigma), \theta, \sigma\right)=$ 0 , so $\frac{\partial \psi_{m}(\theta, \sigma)}{\partial \theta}=-\frac{H_{\psi \theta}\left(\psi_{m}(\theta, \sigma), \theta, \sigma\right)}{H_{\psi \psi}\left(\psi_{m}(\theta, \sigma), \theta, \sigma\right)} . \quad H_{\psi \theta}(\psi, \theta, \sigma)=1+(1-\phi) \beta>0$ and $H_{\psi \psi}(\psi, \theta, \sigma)>0$, so $\frac{\partial \psi_{m}(\theta, \sigma)}{\partial \theta}<0$.

Differentiating $H_{\psi}\left(\psi_{m}(\theta, \sigma), \theta, \sigma\right)=0$ with respect to $\sigma$ yields

${ }^{26}$ To prove that $\psi_{0}(\sigma)<1$, observe that $\psi_{0}(\sigma)-1=$
$[(1-\sigma)(1+\beta)+\sigma \beta \phi]^{-1}[\phi(1-\alpha) \beta+\phi \alpha \beta \sigma-(1-\sigma)(1+\beta)-\sigma \beta \phi]$
$[(1-\sigma)(1+\beta)+\sigma \beta \phi]^{-1}(1-\sigma)[(1-\alpha) \phi \beta-(1+\beta)]<0$.


$H_{\psi \psi}\left(\psi_{m}(\theta, \sigma), \theta, \sigma\right) \frac{\partial \psi_{m}(\theta, \sigma)}{\partial \sigma}+H_{\psi \sigma}\left(\psi_{m}(\theta, \sigma), \theta, \sigma\right)=0$, which implies $\frac{\partial \psi_{m}(\theta, \sigma)}{\partial \sigma}=$ $-\frac{H_{\psi \sigma}\left(\psi_{m}(\theta, \sigma), \theta, \sigma\right)}{H_{\psi \psi}\left(\psi_{m}(\theta, \sigma), \theta, \sigma\right)}$. Observe that if $\theta<\bar{\theta}(\sigma)$, then $H_{\psi \sigma}\left(\psi_{m}(\theta, \sigma), \theta, \sigma\right)=$ $-\frac{1}{1-\alpha} \frac{1+(1-\phi) \beta}{\phi}\left(2 \psi_{m}(\theta, \sigma)-\lambda_{L}^{*}\right)<0$ because $\theta<\bar{\theta}(\sigma)$ implies $\psi_{m}(\theta, \sigma)>0$. Therefore, $\frac{\partial \psi_{m}(\theta, \sigma)}{\partial \sigma}>0$.

Lemma 3 Define $h(\theta, \sigma) \equiv \min _{\psi} H(\psi, \theta, \sigma)=H\left(\psi_{m}(\theta, \sigma), \theta, \sigma\right)$. If $\theta<$ $\bar{\theta}(\sigma)$, then $h(\theta, \sigma)$ is strictly increasing in $\theta$ and strictly decreasing in $\sigma$.

Proof. Assume that $\theta<\bar{\theta}(\sigma)$. Note that $\frac{\partial h(\theta, \sigma)}{\partial \theta}=H_{\psi}\left(\psi_{m}(\theta, \sigma), \theta, \sigma\right) \frac{\partial \psi_{m}(\theta, \sigma)}{\partial \theta}+$ $H_{\theta}\left(\psi_{m}(\theta, \sigma), \theta, \sigma\right)$. Since $\psi_{m}(\theta, \sigma)$ minimizes $H(\psi, \theta, \sigma)$ with respect to $\psi, H_{\psi}\left(\psi_{m}(\theta, \sigma), \theta, \sigma\right)=0$. Lemma 2 implies that $\psi_{m}(\theta, \sigma)>0>\lambda_{L}^{*}$ so statement (3) of Lemma 1 implies that $H_{\theta}\left(\psi_{m}(\theta, \sigma), \theta, \sigma\right)>0$. Therefore, $\frac{\partial h(\theta, \sigma)}{\partial \theta}>0$.

Note that $\frac{\partial h(\theta, \sigma)}{\partial \sigma}=H_{\psi}\left(\psi_{m}(\theta, \sigma), \theta, \sigma\right) \frac{\partial \psi_{m}(\theta, \sigma)}{\partial \sigma}+H_{\sigma}\left(\psi_{m}(\theta, \sigma), \theta, \sigma\right)$. Again, since $\psi_{m}(\theta, \sigma)$ minimizes $H(\psi, \theta, \sigma)$ with respect to $\psi, H_{\psi}\left(\psi_{m}(\theta, \sigma), \theta, \sigma\right)=$ 0 . Lemma 2 implies that $\psi_{m}(\theta, \sigma)>0>\lambda_{L}^{*}$ so statement (4) of Lemma 1 implies that $H_{\sigma}\left(\psi_{m}(\theta, \sigma), \theta, \sigma\right)<0$. Therefore, $\frac{\partial h(\theta, \sigma)}{\partial \sigma}<0$.

Lemma 4 If $H(0, \theta, \sigma)>0$ and $H_{\psi}(0, \theta, \sigma) \geq 0$, then $H(\psi, \theta, \sigma)=0$ has no positive real roots.

Proof. $H_{\psi \psi}(\psi, \theta, \sigma)=2\left[\frac{(1-\sigma)(1+\beta)}{\phi}+\sigma \beta\right] \frac{1}{1-\alpha}>0$ implies that $H_{\psi}(\psi, \theta, \sigma)>$ $H_{\psi}(0, \theta, \sigma) \geq 0$ for all $\psi>0$. Therefore, $H(\psi, \theta, \sigma)>H(0, \theta, \sigma)>0$ for all $\psi>0$.

Corollary 5 If $\theta \geq \bar{\theta}(\sigma)$, then $H(\psi, \theta, \sigma)=0$ has no positive real roots.

Proof. If $\theta \geq \bar{\theta}(\sigma)$, then statements (1) and (2) of Lemma 1 imply $H(0, \theta, \sigma) \geq 0$ and $H_{\psi}(0, \theta, \sigma)>0$. Therefore, Lemma 4 implies that $H(\psi, \theta, \sigma)=0$ has no positive real roots.

Lemma $5 H\left(\lambda_{L}^{*}, \theta, \sigma\right)>0$ and is independent of $\theta$ and $\sigma$, where $\lambda_{L}^{*} \equiv$ $-\frac{\beta \phi \alpha}{1+(1-\phi) \beta}<0$.

Proof. $H\left(\lambda_{L}^{*}, \theta, \sigma\right)=\left[\frac{(1-\sigma)(1+\beta)}{\phi}+\sigma \beta\right] \frac{1}{1-\alpha} \lambda_{L}^{* 2}-\left[\beta+\sigma \frac{\alpha \beta}{1-\alpha}\right] \lambda_{L}^{*}=\frac{1+\beta}{\phi} \frac{1}{1-\alpha} \lambda_{L}^{* 2}-$ $\beta \lambda_{L}^{*}$ is independent of $\theta$ and $\sigma$, and is positive because $\lambda_{L}^{*}<0$. 
Lemma 6 If $\phi>0, \exists$ a unique $\theta_{\max }(\sigma) \in(0, \bar{\theta}(\sigma))$ such that $H\left(\psi, \theta_{\max }(\sigma), \sigma\right)=$ 0 has a (positive) repeated real root, where $\bar{\theta}(\sigma) \equiv \frac{\beta\left(1+\sigma \frac{\alpha}{1-\alpha}\right)}{1+\beta-\beta \phi}>0 . \quad \theta_{\max }(\sigma)$ is strictly increasing in $\sigma$.

Proof. Define $h(\theta, \sigma) \equiv \min _{\psi} H(\psi, \theta, \sigma)=H\left(\psi_{m}(\theta, \sigma), \theta, \sigma\right)$. Since $H(0,0, \sigma)=0$ and $H_{\psi}(0,0, \sigma)=-\left(\beta+\sigma \frac{\alpha \beta}{1-\alpha}\right)<0, h(0, \sigma)<0$. Lemma 2 implies that $\psi_{m}(\bar{\theta}(\sigma), \sigma)=0$. Therefore, $h(\bar{\theta}(\sigma), \sigma)=H(0, \bar{\theta}(\sigma), \sigma)=$ $\beta \bar{\theta} \phi \alpha>0$. Since $h(\theta, \sigma)$ is a continuous function of $\theta, h(0, \sigma)<0<$ $h(\bar{\theta}(\sigma), \sigma)$ implies that there exists a $\theta_{\max }(\sigma) \in(0, \bar{\theta})$ such that $h\left(\theta_{\max }(\sigma), \sigma\right)=$ 0 . Lemma 3 implies that $h(\theta, \sigma)$ is strictly increasing in $\theta$ for $\theta<\bar{\theta}(\sigma)$, so the value of $\theta_{\max }(\sigma) \in(0, \bar{\theta}(\sigma))$ for which $h\left(\theta_{\max }(\sigma), \sigma\right)=0$ is unique. Differentiate $h\left(\theta_{\max }(\sigma), \sigma\right)=0$ with respect to $\sigma$ to obtain $\frac{\partial h\left(\theta_{\max }(\sigma), \sigma\right)}{\partial \theta} \theta_{\max }^{\prime}(\sigma)+$ $\frac{\partial h\left(\theta_{\max }(\sigma), \sigma\right)}{\partial \sigma}=0$. Therefore, $\theta_{\max }^{\prime}(\sigma)=-\frac{\frac{\partial h\left(\theta_{\max }(\sigma), \sigma\right)}{\partial \sigma^{\sigma}\left(\theta_{\max }(\sigma), \sigma\right)}}{\frac{\partial\left(\theta^{2}\right.}{\partial \theta}}$. Since $\theta_{\max }(\sigma)<\bar{\theta}(\sigma)$, Lemma 3 implies $\frac{\partial h\left(\theta_{\max }(\sigma), \sigma\right)}{\partial \theta}>0$ and $\frac{\partial h\left(\theta_{\max }(\sigma), \sigma\right)}{\partial \sigma}<0$, so that $\theta_{\max }^{\prime}(\sigma)>0$.

Corollary 6 If $\theta \leq \theta_{\max }(\sigma)$, then $H(\psi, \theta, \sigma)=0$ has two real roots $\lambda_{L}(\theta, \sigma) \leq$ $\lambda_{H}(\theta, \sigma)$ with the following properties: $\lambda_{H}(\theta, \sigma)>0, \lambda_{L}(\theta, \sigma)>\lambda_{L}^{*}$. If $\theta<$ $\theta_{\max }(\sigma)$, the roots are distinct, $\frac{\partial \lambda_{L}(\theta, \sigma)}{\partial \theta}>0, \frac{\partial \lambda_{H}(\theta, \sigma)}{\partial \theta}<0$, $\operatorname{sign}\left(\frac{\partial \lambda_{L}(\theta, \sigma)}{\partial \sigma}\right)=$ $-\operatorname{sign}\left(\lambda_{L}(\theta, \sigma)\right)$, and $\frac{\partial \lambda_{H}(\theta, \sigma)}{\partial \sigma}>0$.

Proof. Lemma 3 states that $h(\theta, \sigma)$ is strictly increasing in $\theta$ for $\theta<$ $\bar{\theta}(\sigma)$. Since $h\left(\theta_{\max }(\sigma), \sigma\right)=0, h(\theta, \sigma)<0$ for $\theta<\theta_{\max }(\sigma)$. Therefore, $H(\psi, \theta, \sigma)=0$ has a repeated real root if $\theta=\theta_{\max }(\sigma)$ and has two distinct real roots if $\theta<\theta_{\max }(\sigma)$.

To prove that $\lambda_{H}(\theta, \sigma)>0$, use statement (2) of Lemma 1 to infer that $H_{\psi}(0, \theta, \sigma)<0$. Therefore, since $H_{\psi \psi}(\psi, \theta, \sigma)=2\left[\frac{(1-\sigma)(1+\beta)}{\phi}+\sigma \beta\right] \frac{1}{1-\alpha}>$ $0,0<\psi_{m}(\theta, \sigma) \leq \lambda_{H}(\theta, \sigma)$. To prove that $\lambda_{L}(\theta, \sigma)>\lambda_{L}^{*}$, suppose that $\lambda_{L}(\theta, \sigma) \leq \lambda_{L}^{*}$. Since $\lambda_{L}^{*}<0<\lambda_{H}(\theta, \sigma), \lambda_{L}^{*} \in\left[\lambda_{L}(\theta, \sigma), \lambda_{H}(\theta, \sigma)\right]$. $H_{\psi \psi}(\psi, \theta, \sigma)>0$ implies that $H(\psi, \theta, \sigma) \leq 0$ for all $\psi \in\left[\lambda_{L}(\theta, \sigma), \lambda_{H}(\theta, \sigma)\right]$. Therefore, $H\left(\lambda_{L}^{*}, \theta, \sigma\right) \leq 0$, which contradicts Lemma 5. Hence, $\lambda_{L}(\theta, \sigma)>$ $\lambda_{L}^{*}$. To determine the effect of $\theta$ on each of the roots, observe that $H_{\psi \psi}(\psi, \theta, \sigma)>$ 0 implies that if there are two distinct real roots, then $H_{\psi}\left(\lambda_{L}(\theta, \sigma), \theta, \sigma\right)<0$ and $H_{\psi}\left(\lambda_{H}(\theta, \sigma), \theta, \sigma\right)>0$. Statement (3) in Lemma 1 implies that $H_{\theta}\left(\lambda_{L}(\theta, \sigma), \theta, \sigma\right)>0$ and $H_{\theta}\left(\lambda_{H}(\theta, \sigma), \theta, \sigma\right)>0$. Applying the implicit 
function theorem to $H\left(\lambda_{i}(\theta, \sigma), \theta, \sigma\right)=0$ for $i=L, H$ implies $\frac{\partial \lambda_{L}(\theta, \sigma)}{\partial \theta}=$ $-\frac{H_{\theta}\left(\lambda_{L}(\theta, \sigma), \theta, \sigma\right)}{H_{\psi}\left(\lambda_{L}(\theta, \sigma), \theta, \sigma\right)}>0$ and $\frac{\partial \lambda_{H}(\theta, \sigma)}{\partial \theta}=-\frac{H_{\theta}\left(\lambda_{H}(\theta, \sigma), \theta, \sigma\right)}{H_{\psi}\left(\lambda_{H}(\theta, \sigma), \theta, \sigma\right)}<0$. Since $\lambda_{L}(\theta, \sigma)>\lambda_{L}^{*}$, statement (4) in Lemma 1 implies that $\operatorname{sign}\left(H_{\sigma}\left(\lambda_{L}(\theta, \sigma), \theta, \sigma\right)\right)=-\operatorname{sign}\left(\lambda_{L}(\theta, \sigma)\right)$ and $H_{\sigma}\left(\lambda_{H}(\theta, \sigma), \theta, \sigma\right)<0$. The implicit function theorem implies sign $\left(\frac{\partial \lambda_{L}(\theta, \sigma)}{\partial \sigma}\right)=$ $-\operatorname{sign}\left(\frac{H_{\sigma}\left(\lambda_{L}(\theta, \sigma), \theta, \sigma\right)}{H_{\psi}\left(\lambda_{L}(\theta, \sigma), \theta, \sigma\right)}\right)=-\operatorname{sign}\left(\lambda_{L}(\theta, \sigma)\right)$ and $\frac{\partial \lambda_{H}(\theta, \sigma)}{\partial \sigma}=-\frac{H_{\sigma}\left(\lambda_{H}(\theta, \sigma), \theta, \sigma\right)}{H_{\psi}\left(\lambda_{H}(\theta, \sigma), \theta, \sigma\right)}>$ 0 .

Corollary 7 If $\theta>\theta_{\max }(\sigma)$, then $H(\psi, \theta, \sigma)=0$ has no real roots.

Proof. The proof of Lemma 6 states that $h\left(\theta_{\max }(\sigma), \sigma\right)=0$ and Lemma 3 states that $h(\theta, \sigma)$ is strictly increasing in $\theta$ for $\theta<\bar{\theta}(\sigma)$. Therefore, $h(\theta, \sigma)>0$ for $\theta_{\max }(\sigma)<\theta<\bar{\theta}(\sigma)$ and $H(\psi, \theta, \sigma)=0$ has no real roots for $\theta_{\max }(\sigma)<\theta<\bar{\theta}(\sigma)$. Corollary 5 states that $H(\psi, \theta, \sigma)=0$ has no real roots for $\theta>\bar{\theta}(\sigma)$.

Lemma 7 Define $\theta_{\min }(\sigma) \equiv-\frac{1}{\phi} \frac{1-\sigma}{1-\alpha}<0$. Then $\lambda_{H}\left(\theta_{\min }(\sigma), \sigma\right)=1$ and $\lambda_{L}^{*}<\lambda_{L}\left(\theta_{\min }(\sigma), \sigma\right)<0$.

Proof. It is straightforward to show that $H\left(1,-\frac{1}{\phi} \frac{1-\sigma}{1-\alpha}, \sigma\right)=0$. Statement (1) of Lemma 1 implies that $H\left(0, \theta_{\min }(\sigma), \sigma\right)<0$ which implies that $\lambda_{L}\left(\theta_{\min }(\sigma), \sigma\right)<0$. Corollary 6 implies that $\lambda_{L}^{*}<\lambda_{L}\left(\theta_{\min }(\sigma), \sigma\right)$.

The following lemma summarizes the values of the roots for various values of $\theta$.

Lemma 8 Suppose that $0<\phi \leq 1$. Define $\bar{\theta}(\sigma) \equiv \frac{\beta\left(1+\sigma \frac{\alpha}{1-\alpha}\right)}{1+\beta-\beta \phi}>0, \lambda_{L}^{*} \equiv$ $-\frac{\beta \phi \alpha}{1+(1-\phi) \beta}<0, \theta_{\min }(\sigma) \equiv-\frac{1}{\phi} \frac{1-\sigma}{1-\alpha}<0$, and $0<\psi_{0}(\sigma) \equiv \phi \frac{(1-\alpha) \beta+\sigma \alpha \beta}{(1-\sigma)(1+\beta)+\sigma \beta \phi}<$ 1. $\exists$ a unique $\theta_{\max }(\sigma) \in(0, \bar{\theta}(\sigma))$ such that $H\left(\psi, \theta_{\max }(\sigma), \sigma\right)=0$ has a (positive) repeated real root, and $\theta_{\max }(\sigma)$ is strictly increasing in $\sigma$. The roots $\lambda_{L}(\theta, \sigma) \leq \lambda_{H}(\theta, \sigma)$ of $H(\psi, \theta, \sigma)=0$ depend on $\theta$ as follows:

(1) If $\theta>\theta_{\max }(\sigma)>0$, then $H(\psi, \theta, \sigma)=0$ has no positive real roots.

(2) If $\theta=\theta_{\max }(\sigma)>0$, then $0<\lambda_{L}(\theta, \sigma)=\lambda_{H}(\theta, \sigma)<\lambda_{H}(0, \sigma)=$ $\psi_{0}(\sigma)<1$.

(3) If $0<\theta<\theta_{\max }(\sigma)$, then $0<\lambda_{L}(\theta, \sigma)<\lambda_{H}(\theta, \sigma)<\lambda_{H}(0, \sigma)=$ $\psi_{0}(\sigma)<1$.

(4) If $\theta=0$, then $\lambda_{L}(\theta, \sigma)=0$ and $0<\lambda_{H}(\theta, \sigma)=\psi_{0}(\sigma)<1$.

(5) If $\theta_{\min }(\sigma)<\theta<0$, then $\lambda_{L}^{*}<\lambda_{L}(\theta, \sigma)<0<\psi_{0}(\sigma)<\lambda_{H}(\theta, \sigma)<1$.

(6) If $\theta=\theta_{\min }(\sigma)$, then $\lambda_{L}^{*}<\lambda_{L}(\theta, \sigma)<0$ and $\lambda_{H}(\theta, \sigma)=1$.

(7) If $\theta<\theta_{\min }(\sigma)$, then $\lambda_{L}^{*}<\lambda_{L}(\theta, \sigma)<0$ and $\lambda_{H}(\theta, \sigma)>1$. 
Lemma 9 Suppose that $x_{t}=f\left(x_{t-1}\right) \equiv \frac{a x_{t-1}+b}{d x_{t-1}+e}$ where $d>0, a+e>0$ and be $\leq 0$. The positive steady-state values of $x$ (for which $x_{t}=x_{t-1}=x$ ), if they exist, are given by the positive real roots of $Q(x) \equiv d x^{2}+e x-a x-b=0$. Suppose that $Q(x)=0$ has two distinct real roots $\lambda_{L}<\lambda_{H}$. If $\lambda_{H}>0$, it is locally stable. If $\lambda_{L}>0$, it is locally unstable.

Proof. The roots $\lambda_{L}$ and $\lambda_{H}$ satisfy $Q\left(\lambda_{i}\right)=d \lambda_{i}^{2}+e \lambda_{i}-a \lambda_{i}-b=0$ for $i=$ $L, H . \quad Q^{\prime \prime}=2 d>0$ implies that $Q^{\prime}\left(\lambda_{L}\right)<0<Q^{\prime}\left(\lambda_{H}\right)$. Confine attention to positive roots $\lambda_{i}>0$. First prove, by contradiction that $d \lambda_{i}+e>0$. Assume that $d \lambda_{i}+e \leq 0$, which implies that $e<0$ because $d>0$ and $\lambda_{i}>0$. Since $e<0, a+e>0$ implies $a>0$, and $b e \leq 0$ implies $b \geq 0$. Therefore, $a \lambda_{i}+b>0$. But $Q\left(\lambda_{i}\right)=0$ implies $\left(d \lambda_{i}+e\right) \lambda_{i}=a \lambda_{i}+b>0$, which contradicts $d \lambda_{i}+e \leq 0$ for $\lambda_{i}>0$. Therefore, $d \lambda_{i}+e>0$. Therefore, $\lambda_{i}=\frac{a \lambda_{i}+b}{d \lambda_{i}+e}=f\left(\lambda_{i}\right)$, so $x_{t}=x_{t-1}=\lambda_{i}$ satisfies the equation of motion for $x_{t}$. Observe that $f^{\prime}\left(\lambda_{i}\right)=\frac{a}{d \lambda_{i}+e}-d \frac{a \lambda_{i}+b}{\left(d \lambda_{i}+e\right)^{2}}$. Use $\lambda_{i}=\frac{a \lambda_{i}+b}{d \lambda_{i}+e}$ to rewrite the second term on the right hand side and simplify to obtain $f^{\prime}\left(\lambda_{i}\right)=\frac{a-d \lambda_{i}}{d \lambda_{i}+e}$, which implies that $f^{\prime}\left(\lambda_{i}\right)-1=-\frac{2 d \lambda_{i}+e-a}{d \lambda_{i}+e}=-\frac{Q^{\prime}\left(\lambda_{i}\right)}{d \lambda_{i}+e}$. If $\lambda_{H}>0$, then $d \lambda_{H}+e>0$, which, along with $Q^{\prime}\left(\lambda_{H}\right)>0$, implies $f^{\prime}\left(\lambda_{H}\right)-1<0$. Also, note that $f^{\prime}\left(\lambda_{i}\right)+1=\frac{a+e}{d \lambda_{i}+e}>0$. Therefore, $-1<f^{\prime}\left(\lambda_{H}\right)<1$, so $\lambda_{H}$ is locally stable. If $\lambda_{L}>0$, then $d \lambda_{L}+e>0$, which, along with $Q^{\prime}\left(\lambda_{L}\right)<0$, implies $f^{\prime}\left(\lambda_{L}\right)-1>0$. Therefore, $f^{\prime}\left(\lambda_{L}\right)>1$, so $\lambda_{L}$ is locally unstable. 


\section{References}

[1] Abel, Andrew B., "On the Invariance of the Rate of Return to Convex Adjustment Costs," Review of Economic Dynamics, 5, 3 (July 2002), 586-601.

[2] Abel, Andrew B., "Will Bequests Attenuate the Predicted Meltdown in Stock Prices When Baby Boomers Retire?"

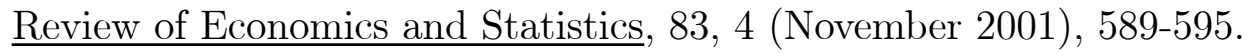

[3] Abel, Andrew B., Mankiw, N. Gregory, Summers, Lawrence H., and Zeckhauser, Richard J., "Assessing Dynamic Efficiency: Theory and Evidence," Review of Economic Studies, 56 (1989), 1-20.

[4] Basu, Parantap, "An Adjustment Cost Model of Asset Pricing," International Economic Review, 28, 3 (October 1987), 609-621

[5] Bohn, Henning, "Social Security and Demographic Uncertainty: The Risk Sharing Properties of Alternative Policies," National Bureau of Economic Research Working Paper No. 7030, March 1999.

[6] Diamond, Peter A., "National Debt in a Neoclassical Growth Model," American Economic Review, 55, 5 (December 1965), 1126-1150.

[7] Hall, Robert E., "The Stock Market and Capital Accumulation," American Economic Review, 91, 5 (December 2001), 1185-1202.

[8] Lucas, Robert E., Jr., "Asset Prices in an Exchange Economy," Econometrica, 46 (1978) 1429-1445.

[9] Zilcha, Itzhak, "Characterizing Efficiency in Stochastic Overlapping Generations Models," Journal of Economic Theory, 55, 1 (October 1991), 116. 